\title{
INVESTIGACIONES
}

\section{Estado del arte sobre inserción de niños y niñas migrantes en el sistema escolar chileno*}

\author{
The state of the art on the insertion of migrant children \\ in the Chilean school system
}

\author{
Carolina Stefoni ${ }^{a, b}$, Javier Corvalán ${ }^{c}$ \\ ${ }^{a}$ Centro Sociedad Tecnológica y Futuro Humano, Universidad Mayor, Chile. \\ ${ }^{b}$ Investigadora asociada Centro Estudios del Conflicto y Cohesión Social, COES. \\ cstefoni@gmail.com \\ ${ }^{c}$ Facultad de Educación. Universidad Alberto Hurtado. \\ jcorvala@uahurtado.cl
}

\begin{abstract}
RESUMEN
El artículo tiene por objetivo realizar un estado del arte sobre la investigación llevada a cabo en Chile respecto de la situación de niños y niñas migrantes en el sistema escolar. A partir de una revisión de publicaciones académicas se analiza cómo se configura este campo de investigación, qué preguntas están planteando los y las investigadores, qué conceptos y categorías son utilizadas, y qué problemas son relevados y puntualizados.

La búsqueda se concentró entre 2005 y 2017. Se seleccionaron dos tipos de textos, por una parte, aquellos de carácter científico (artículos en revistas y capítulos de libros) que abordaron en específico el tema de migración y educación en el contexto nacional -32 textos en total-, y por otra, documentos oficiales emanados del Ministerio de Educación relacionados con migración y educación escolar (normativas, recomendaciones técnicas) así como guías para una educación inclusiva en contextos migratorios.
\end{abstract}

Palabras claves: Educación, migración, interculturalidad.

\section{ABSTRACT}

The article aims to make a state of the art focused on the research carried out in Chile regarding the situation of migrant children in the school system. Through the review of academic publications, the article analyze how this research field is configured, what questions are being posed by the researchers, what concepts and categories are used and what problems are relieved and pointed out.

The search was concentrated between 2005 and 2017. Two types of texts were selected, on the one hand, those of a scientific nature (articles and book chapters) specific to migration and education focused on the national context -32 texts in total-, and another, official documents emanating from the Ministry of Education related to migration and school education (regulations, technical recommendations) and guidelines for inclusive education in migratory contexts.

Key words: education, migration, interculturalism.

\footnotetext{
* Este capítulo fue posible gracias al financiamiento de FONIDE FX11622-2016 "Construyendo escuelas interculturales: elaboración participativa de una hoja de ruta para asistentes de la educación, profesores y directivos" y también por el Centro de Estudios de Conflicto y Cohesión Social - COES, Proyecto CONICYT/FONDAP/15130009. Las opiniones que se presentan en esta publicación, así como los análisis e interpretaciones, son de exclusiva responsabilidad de los autores y no reflejan necesariamente la opinión del MINEDUC.
} 


\section{INTRODUCCIÓN}

América del Sur ha experimentado en las últimas décadas un significativo crecimiento de la inmigración, llegando a contar con 4.7 millones de personas inmigrantes lo que corresponde al 1.2\% de la población total regional (Texidó \& Gurrieri, 2012). Algunas de las razones que explican este crecimiento son las mayores restricciones para el ingreso a Estados Unidos -lo que ha generado un redireccionamiento de los flujos de personas hacia otros destinos-; la crisis económica en España, que llevó a que se redujera el ingreso de migrantes latinoamericanos hacia ese país después de una década de crecimiento migratorio sostenido (Cerruti, Maguid \& Díaz Gil, 2011); las mayores facilidades comparativas que presenta para los latinoamericanos migrar a países de la región en el contexto de los procesos de integración y del acuerdo de residencia de los países del Mercosur implementado en 2009 (Ceriani, 2011); y la particular situación de Venezuela en los últimos años, que se ha traducido en la salida de cerca de 3 millones de personas, muchos de los cuales se dirigen a otros países de América Latina. A todo lo anterior se agregan los menores costos asociados en relación a destinos más lejanos como Europa y Estados Unidos.

En este escenario, Chile destaca como el país donde más ha crecido la inmigración en los últimos años. Este crecimiento sostenido se refleja en cerca de un millón de personas extranjeras que han llegado al país atraídas por una estabilidad política, social y económica (Guizardi, Moraga \& Garcés, 2014; Stefoni, 2011). Si bien todavía no se cuenta con un número definido de cuántos son los migrantes en el país, las proyecciones oficiales plantean cifras de entre 700.000 y un millón de personas (Rojas \& Silva, 2016). Tal crecimiento se vuelve realidad en cada uno de los ámbitos de la sociedad, cuestión que plantea importantes desafíos no sólo a la política pública sectorial, sino que obliga a volver sobre los principios de igualdad, ciudadanía y derechos humanos, de modo de reforzar la condición de universalidad de estos.

En este capítulo realizaremos un estado del arte respecto de la investigación científica en una de las dimensiones que más atención ha capturado en los últimos años: el sistema escolar y la realidad de niños y niñas migrantes. Se trata de un campo de estudio que se ha desarrollado de la mano del incremento de la matrícula de niños extranjeros, con una importante concentración en las escuelas municipales, tal como lo detalla la tabla $\mathrm{N}^{\mathrm{o}} 1$. La información entregada por el Ministerio de Educación muestra un importante incremento de la matrícula de niños extranjeros entre 2015 y 2016 (incremento de un 99.5\%), llegando a representar el $1,7 \%$ del total de niños y niñas matriculados en el sistema escolar. Las cifras para el 2017 muestran un nuevo incremento, pero a un ritmo menor que el año anterior (27\%). La concentración en colegios municipales ha sido sostenida en los últimos tres años (61\% en 2015; 55\% en 2016; $57 \%$ en 2017).

La alta concentración de migrantes en determinadas regiones y comunas, así como la mayor presencia que tienen en establecimientos municipales (gratuitos) tiene como consecuencia la existencia de colegios con altos porcentajes de matrícula de niños extranjeros (tasas superiores al 50\%). Esta alta concentración introduce la variable migración en el análisis sobre las formas de reproducción de la desigualdad en el sistema escolar. El trabajo de Sleeter, Montecinos y Jiménez (2016) es clave en este sentido, por cuanto es una de las pocas reflexiones que se detiene a analizar la incorporación de los niños y niñas migrantes en un sistema altamente segregado producto de las políticas de privatización de la educación impulsadas en los años ochenta y que han sustentado desde ese entonces al sistema escolar (Sleeter, Montecinos \& Jiménez, 2016). Un segundo 
estudio que contribuye en esta línea es el de Joiko y Vásquez (2016), en donde analizan las preferencias y opciones de establecimientos por parte de familias migrantes para matricular a sus hijos en el sistema escolar chileno (Joiko y Vásquez, 2016).

Tabla 1: Incremento de matrícula de niños, niñas y adolescentes migrantes en el sistema escolar chileno

\begin{tabular}{|l|c|c|c|c|c|}
\hline Dependencia & $\mathbf{2 0 1 5}$ & $\mathbf{2 0 1 6}$ & $\mathbf{2 0 1 7}$ & $\begin{array}{c}\text { Variación } \\
\mathbf{2 0 1 5 - 2 0 1 6}\end{array}$ & $\begin{array}{c}\text { Variación } \\
\mathbf{2 0 1 6 - 2 0 1 7}\end{array}$ \\
\hline Municipal & 18.751 & 33.613 & 44.595 & $79,3 \%$ & $32,7 \%$ \\
\hline Particular subvencionado & 9.914 & 20.387 & 25.711 & $105,6 \%$ & $26,1 \%$ \\
\hline Particular pagado & 1.665 & 6.160 & 6.142 & $270 \%$ & $-0,3 \%$ \\
\hline Corp. de Adm. Delegada & 295 & 925 & 1.159 & $213 \%$ & $25,3 \%$ \\
\hline Total & 30.625 & 61.085 & 77.607 & $99,5 \%$ & $27,0 \%$ \\
\hline
\end{tabular}

Fuente. Centro de estudios, División de Planificación y presupuesto, MINEDUC. Información presentada en taller "Encuentro para la inclusión de estudiantes extranjeros" (11.01.2018).

A través de la realización de este estado del arte buscamos responder de este modo a las siguientes preguntas: ¿cómo se configura este campo de investigación?, ¿cuáles son las preguntas que los investigadores están planteando?, ¿qué conceptos y categorías son utilizadas y qué problemas son relevados y puntualizados? Para llevarlo a cabo se realizó una búsqueda de publicaciones académicas y se seleccionaron aquellos documentos que abordaran la situación de niños y niñas migrantes en el sistema escolar chileno.

El artículo comienza con algunas puntualizaciones de lo que es un estado del arte y la metodología utilizada para su elaboración. Luego entrega los resultados en donde se revisan las aproximaciones metodológicas utilizadas en las investigaciones, y a continuación los cuatro temas que reúnen las reflexiones y conclusiones de los estudios sistematizados. Estos temas son: el derecho a la educación, las representaciones sobre migrantes y migración que tienen diversos actores de la educación, las posibilidades y avances en la construcción de escuelas interculturales y las discusiones sobre racismo presentes en el sistema escolar. Si bien buscamos ordenar la información a partir del corpus formado por todos los documentos y publicaciones revisadas, hemos decidido incluir en distintos momentos nuestras propias reflexiones que derivan de la investigación "Construyendo escuelas interculturales: elaboración participativa de una hoja de ruta para asistentes de la educación, profesores y directivos" (Proyecto FONIDE Nº11622, del 2017).

\section{ESTADO DEL ARTE: ASPECTOS METODOLÓGICOS PARA SU CONSTRUCCIÓN}

De acuerdo con Londoño, Maldonado y Calderón (2014) un estado del arte debe ser capaz de identificar qué problemas asociados al tema de interés se han investigado, cómo se definen estos problemas, qué evidencia empírica se utiliza en el análisis, qué tipo de metodologías se emplean y cuáles son los principales productos de las investigaciones 
realizadas (Londoño, Maldonado \& Calderón, 2014). Esto supone al menos dos momentos; primero, una sistematización de lo investigado, es decir, organizar el corpus de acuerdo a las preguntas de investigación, metodologías y/o resultados obtenidos, y segundo, una instancia en la que se puedan relacionar los distintos trabajos e identificar áreas, sub campos, enfoques epistemológicos, así como vacíos y redundancias que se producen. En la sección que sigue se presenta la síntesis de ambas dimensiones, esto es la identificación de los principales temas y reflexiones que han sido trabajados por investigadores, y reflexión sobre los supuestos, conceptos y categorías utilizados. En las reflexiones finales se abordarán algunas conclusiones y proyecciones de temas en este campo de investigación, enfatizando ciertas ausencias que pudimos detectar en esta revisión.

En términos de alcance y cobertura, se revisó la producción académica sobre educación y migración en Chile, publicada en revistas nacionales e internacionales. Para la búsqueda de artículos y publicaciones se utilizaron descriptores como educación y migración, niños y niñas migrantes, educación intercultural y migraciones, políticas migratorias en educación, entre otros. Dado que se trata de un campo de reciente desarrollo, se incluyeron textos que dan cuenta de la situación de niños y niñas migrantes, no necesariamente vinculados a la experiencia escolar, pero sí desde la perspectiva de la infancia (Pavéz, 2012; 2013).

La búsqueda se realizó a través de buscadores como Scielo, Academia.edu, researchgate.net y revistas especializadas como Estudios Pedagógicos, Polis, Convergencia, Revista de Ciencias Sociales, Revista Electrónica de Investigación Educativa, Revista Mexicana de Investigación Educativa, Psicoperspectivas: Individuo y Sociedad, Revista Latinoamericana de Ciencias Sociales, Niñez y Juventud, Revista Latinoamericana de Educación Inclusiva, Revista Calidad en la Educación, entre otras. En cada uno de los artículos seleccionados se revisó la bibliografía citada de modo de identificar nuevas publicaciones sobre la realidad chilena en esta materia. Las publicaciones aparecieron en su mayoría desde el 2005 hasta diciembre 2017. Con todo, asumimos la responsabilidad de omisiones involuntarias, producto de las dificultades para acceder a revistas cerradas o bien porque fueron publicadas en otras revistas no necesariamente especializadas en educación.

Se seleccionaron dos tipos de textos, por una parte, aquellos de carácter científico (artículos y capítulos de libros) específicos en migración y educación enfocados en el contexto nacional -32 textos en total-, y por otra, documentos oficiales emanados del Ministerio de Educación relacionados con migración y educación escolar (normativas, recomendaciones técnicas) y guías para una educación inclusiva en contextos migratorios. Los textos se ficharon utilizando una pauta que incluyó: título, autores, lugar de publicación, año, objetivos, metodología y principales resultados. No se consideraron tesis de pre o posgrado por contar con acceso restringido a ellos, situación que no garantizaba una adecuada representatividad. Recortes de prensa también quedaron fuera por ser de una naturaleza distinta a los trabajos académicos.

\section{RESULTADOS}

\subsection{EL MAYOR PESO DE LA INVESTIGACIÓN CUALITATIVA}

La mayoría de los estudios revisados utiliza metodologías cualitativas con técnicas como entrevistas, grupos focales, observaciones participante y no participante. En algunos casos 
se trata de aproximaciones de carácter más bien teórico (Barrios \& Palau, 2014; Carrillo, 2014) o bien de análisis documentales a partir de textos determinados (Jiménez, Aguilera, Valdés \& Hernández, 2017). Un número significativo de investigaciones responden a estudios de caso en establecimientos educacionales específicos, la mayoría de ellos en la Región Metropolitana y en algunas regiones del norte del país como Arica y Parinacota, Tarapacá y Antofagasta (Bustos \& Garin, 2017b; Marín, 2014), y en el sur como por ejemplo las regiones del Bio-Bio y de la Araucanía (Sánchez, Navas \& Holgado, 2013). Son pocos los estudios que han incorporado más de una región para la selección de muestras (Joiko \& Vásquez, 2016). En el caso de Santiago, los establecimientos seleccionados se concentraron en aquellas comunas con alto porcentaje de migración (Santiago Centro, Recoleta, Independencia, Quilicura), lo que significa que la mayoría de los casos investigados tiene un alto porcentaje de alumnos inmigrantes (Alvites \& Jiménez, 2011; Cerón, Alvarado \& Poblete, 2017; Donoso, Mardones \& Contreras, 2009; Hernández, 2016; Jiménez \& Fardella, 2015; Moreno \& Oyarzún, 2013; Pavéz, 2017; Poblete \& Galaz, 2016; Salas, Del Río, Kong \& San Martín, 2016; Tijoux, 2013). Estas opciones metodológicas dejan fuera aquellos establecimientos con un número menor de estudiantes extranjeros.

Si bien la selección de casos resulta válida para observar la realidad en establecimientos con alta presencia de niños y niñas extranjeros, sostenemos la importancia de diversificar los criterios de selección de las muestras, de modo de conocer, comprender y eventualmente comparar distintas realidades a lo largo del país. Algunos nuevos criterios posibles de introducir son: tipo de administración de establecimientos (municipal, subvencionado y privados), regiones, sectores urbanos y rurales, colegios de hombres, de mujeres y mixtos, colegios conocidos como "de colonias", establecimientos de distintos grupos socioeconómicos, y establecimientos con alta, media y baja cantidad de migrantes. Esto permitiría entre otras cosas identificar de qué modo el sistema escolar nacional está abordando la diversidad cultural que se instala en el país. Este punto es de gran relevancia ya que la concentración de estudios en establecimientos municipales puede contribuir a situar los desafíos de la migración sólo en dicho sector, sin embargo, la multiculturalidad que comienza a visibilizarse en todo el país requiere de abordajes nacionales que incluyan a todos los tipos de establecimientos.

El crecimiento de la migración es innegable, pero es importante destacar también la necesidad de una mayor reflexión en torno a la producción de estadísticas con que cuenta el país. Tal como señalan Joiko y Vásquez (2016) resulta extremadamente difícil realizar un diagnóstico acabado sobre la situación de los niños y niñas migrantes debido a la poca información y sistematización de esta. En su artículo, las autoras citadas elaboran una breve caracterización con información para el 2016 en la que destacan que el 59\% de los niños y niñas migrantes se encuentra en educación básica y el 23\% en media. Agregan que el 82\% de los estudiantes se concentran en tres regiones: Metropolitana, Antofagasta y Tarapacá, y que si los migrantes se concentran en mayor medida en la educación municipal (55\% del total de niños y niñas migrantes), los nacionales lo hacen en los colegios particulares subvencionados (56\%). Agregan también que de aquellos niños y niñas migrantes que se encuentran en el sistema subvencionado, $70 \%$ asiste a establecimientos gratuitos. Sin embargo, resulta evidente la necesidad de contar con mayor información de modo de poder realizar seguimiento a esta caracterización, observar diferencias por colectivos de migrantes, por años de residencia, por estatus migratorio, por capital social de sus familias, entre otras dimensiones y variables. 


\subsection{PRINCIPALES TEMAS DE INVESTIGACIÓN}

Las investigaciones revisadas abordan distintos aspectos y temas asociados a la educación y migración. En varios casos una misma investigación puede abordar más de un tema, por lo que la selección que aquí presentamos pretende responder a las preguntas señaladas en un comienzo respecto de qué problemas y reflexiones son relevados por los investigadores y qué tipo de conocimiento se está desarrollando en este naciente campo de investigación.

\subsubsection{Derecho a la educación de niños, niñas y adolescentes migrantes}

Un primer tema que concita el interés de los investigadores es la pregunta respecto de si el Estado garantiza efectivamente el derecho a la educación a todos los niños inmigrantes (Contreras, Cortés \& Fabio, 2012; Joiko \& Vásquez, 2016; Pavéz, 2013; Poblete \& Galaz, 2016; 2017; Stefoni, Acosta, Gaymer \& Casas-Cordero, 2010). Estos trabajos diagnosticaron una serie de condiciones que dificultaban el acceso al sistema escolar, poniendo el riesgo el derecho básico a la educación. Entre algunas de las dificultades señaladas (Poblete \& Galaz, 2017) se encuentran la situación de irregularidad de las familias y por ende de los niños y niñas, dificultad para conseguir documentación solicitada por el Estado chileno para regularizar la residencia, ausencia de documentación escolar del país de origen (y costos asociados para su obtención) y dificultad para realizar el proceso de convalidación de estudios (sea por ausencia de documentos, o por provenir de un país con el que no hay convenio de validación). La evidencia de que el Estado no estaba asegurando el derecho a la educación de todos y todas los niños y niñas, independiente de su género, raza, étnica, nacionalidad y estatus migratorio, generó importantes reacciones por parte de las autoridades, lo que permitió desarrollar una serie de instrumentos para garantizar este derecho. Sin embargo, el camino de la política pública en esta materia ha sido resolver problemas en la medida en que aparecen, a través de circulares, programas especiales, decretos, pero muy pocas leyes. El primer instrumento en esta línea fue el Ordinario 07/1008 (2005) del Ministerio de Educación que instruye sobre el ingreso, permanencia y ejercicio de los derechos de los alumnos/as inmigrantes en los establecimientos educacionales. Este ordinario no fue suficiente para garantizar el acceso y permanencia de los estudiantes, pero lo más importante es que quedó en evidencia la necesidad de contar con instrumentos más generales que permitieran encuadrar las distintas medidas necesarias de seguir adoptando. Así, actualmente se reconocen dos instrumentos de carácter más general que establecen la obligación por parte del Estado de garantizar el derecho a la educación: el Decreto 1393 (2014), que crea el Consejo Nacional de Política Migratoria, cuya tercera instrucción refiere a niños y niñas inmigrantes y educación, y el Instructivo Presidencial No 5 (2015), que garantiza a niños, niñas y adolescentes de familias migrantes el acceso, permanencia y progreso de su trayectoria educativa en igualdad de condiciones que todos los niños (MINEDUC. División de Educación General, Coordinación Nacional de Inclusión y Diversidad, 2017).

Las investigaciones más recientes han detectado nuevas dificultades que enfrentan los niños y niñas migrantes para ejercer el derecho a la educación. Por ejemplo, si bien podían asistir a los establecimientos educacionales, no podían ser promovidos de curso si su situación migratoria no era regularizada por sus padres, no podían acceder a la subvención escolar preferencial ni a otras becas o recursos, y continuaban teniendo problemas en el reconocimiento legal de su nivel escolar. La detección de estos vacíos y el trabajo de las 
organizaciones sociales contribuyó a que se establecieran una serie de ordinarios con el objetivo de resolver estas situaciones. Destacan en este punto el Ordinario 329/2017 (que complementa el ordinario 894/2016) y que estableció la entrega por parte del Ministerio de Educación del Identificador Provisorio Escolar (IPE), un número único que se entrega a todos los estudiantes que no contaban con un número de identificación nacional definitivo (RUT), independiente a su estatus migratorio. El IPE reemplazó al antiguo "Rut100", otro identificador asignado a los estudiantes que los dejaba en una situación ambigua cuando sus padres no lograban regularizar su situación administrativa. El segundo ordinario es el 894/2016 que actualizó el instructivo sobre ingreso, permanencia y ejercicio de los derechos de estudiantes migrantes en establecimientos escolares (ordinario 1008/2005). Este ordinario estableció una matrícula provisoria asociada al IPE y que será cambiada por una matrícula definitiva en cuanto concluya el proceso de validación de estudios. Respecto de la validación y convalidación de estudios, se dictaron los ordinarios 894 y 329 en donde se especifican las condiciones y procedimientos a seguir para este proceso.

\subsubsection{Representaciones, estereotipos y actitudes de actores del sistema escolar frente a la presencia de niños, niñas y adolescentes migrantes}

La presencia de niños y niñas migrantes en la sala de clases ha llevado a diversos investigadores a analizar cuáles son las representaciones que tienen profesores y equipos directivos respecto de ellos. La pregunta es clave ya que, tal como lo manifiestan diversos investigadores, las representaciones que tengan los profesores respecto de los alumnos es una variable decisiva en el proceso de enseñanza y en la construcción de una escuela inclusiva, tarea que en Chile ha adquirido una significación aún mayor a partir de la entrada en vigencia de la nueva ley de inclusión escolar.

"Es claro que la importancia de las percepciones que tengan los docentes respecto de la migración está en el hecho que de estas dependerán no solo el trato que otorguen a los/ as estudiantes o su rendimiento (Jordán, 1994; León, Mira y Gómez, 2007), sino también la posibilidad de aportar a una cultura inclusiva al interior de las escuelas, entendida como una comunidad que promueve valores coherentes con los principios de la democracia, solidaridad y el respeto irrestricto a la diversidad (Blanco, 1999, 2005; Booth y Ainscow, 2000; Echeita, 2008)" (Cerón, Alvarado \& Poblete, 2017, p. 235).

Este tema es abordado en 8 publicaciones utilizando metodologías cuantitativas, cualitativas y mixtas. Dos de estos estudios son de carácter teórico (Bravo, 2012; Carrillo, 2014), tres se realizaron en Santiago (Cerón et al., 2017; Moreno \& Oyarzún, 2013; Salas, Kong \& Gazmuri, 2017) y tres en regiones (Arica y Parinacota, del Bio-Bio y Araucanía) (Bustos \& Gairin, 2017a, 2017b; Sánchez et al., 2013). El estudio de Sánchez, Navas y Holgado (2013) analiza las actitudes hacia la inmigración de un grupo de estudiantes de pedagogía de las regiones del Bío Bío y de la Araucanía (720 estudiantes en total). El estudio utilizó la Escala de Actitudes hacia la Inmigración de León, Mira y Gómez (Sánchez, Navas \& Holgado, 2013, p. 24) y una de las conclusiones es la presencia de una actitud intermedia, una suerte de discriminación enmascarada que se caracterizaría por evitar relaciones íntimas con los migrantes y por una falta de sentimientos positivos hacia ellos. Esto es de particular importancia, dado que los encuestados son futuros profesores.

El trabajo de Salas, del Río, Kong y San Martín (2016) utiliza el Test de Asociación implícita (IAT) para medir los prejuicios hacia la inmigración de profesores y estudiantes, 
una escala de actitudes hacia la multiculturalidad y un test de autoestima escolar, de modo de observar la relación entre los prejuicios y la autoestima de los niños y niñas nacionales y migrantes. El estudio concluye que, del total de estudiantes que contestaron el instrumento (779 chilenos correspondiente al $72.6 \%$ de la muestra y 294 extranjeros, correspondientes al 27.4\%), el 69\% declara sentirse chileno ya sea por el componente geográfico (haber nacido en el territorio) o por el cultural (lengua, costumbres, tradiciones). En el caso de los profesores, el estudio indica que a mayor distancia con el fenómeno, mejor actitud, y entre un 25 y un $30 \%$ de los entrevistados tiene una valoración negativa hacia la diversidad étnica y presencia de estudiantes inmigrantes en la sala de clases (Salas et al., 2016).

En una línea similar, Cerón, Pérez y Poblete (2017) analizan la percepción que tienen profesores respecto de la inclusión (educativa) de estudiantes migrantes en escuelas de la comuna de Santiago, y las posibilidades que se vislumbran para la construcción de una cultura inclusiva. Los resultados indican que los docentes tienen una visión restringida de la inclusión, y asumen que los estudiantes migrantes tienen una serie de déficit académicos y de comportamiento que dificultan su proceso de inclusión y su plena participación en las escuelas (Cerón et al., 2017).

La idea (por parte de los docentes) de que los niños inmigrantes presentan déficits de algún tipo es reiterada en los estudios de carácter cualitativo. La investigación de Moreno y Oyarzún (Moreno \& Oyarzún, 2013) da cuenta de una baja valoración de la presencia de niños inmigrantes por parte de los docentes, así como de la idea de que tendrían una menor capacidad intelectual, y que las condiciones de vida a las que estarían expuestos generan una serie de déficits que los dejan en una situación de desventaja respecto de sus compañeros.

El trabajo de Bustos y Garín permite matizar estas impresiones. El estudio realizado en un establecimiento rural, en la zona fronteriza de Arica y Parinacota, plantea que habría una mejor valoración hacia los estudiantes extranjeros por parte de los docentes, debido al mejor desempeño académico que ellos muestran. Es interesante precisar que en este caso los apoderados, si bien valoran el acceso que tienen sus hijos a la educación, consideran que la formación que están recibiendo es de menor calidad que la que se les brindaba en sus países de origen.

Ahora bien, independiente de la valoración que tiene la migración (positiva o negativa), en todos los casos estudiados los autores señalan que predomina el discurso asimilacionista de la escuela hacia los niños y niñas migrantes, es decir, por una parte, recae en los propios sujetos la obligación de adaptarse a la cultura nacional y, por otra parte, su presencia no logra alterar las formas de enseñanza o la cultura institucional. Una cita extraída del estudio de Bustos y Garín lo ejemplifica claramente:

En los contextos rurales, se percibe la normalización y la homogeneización cultural como su principal tarea: 'se les apoya en los trámites de residencia, y para que saquen el RUT chileno. (...) Lo que sí hay que tratar de imponer nuestros valores y para ello aprovechamos los actos cívicos del lunes'. (Bustos \& Garin, 2017a).

El carácter asimilacionista que prima en los establecimientos educacionales es un dato extremadamente relevante, por cuanto lleva a preguntarnos sobre las posibilidades de transformación del sistema escolar que vayan más allá de la adopción de los discursos de la inclusión. Un tema relevante en este contexto refiere a la formación docente y formación continua en esta materia, que abordan Sánchez y Navas (Sánchez et al., 2013). 


\subsubsection{Escuelas interculturales: la distancia entre teoría y práctica}

Los resultados en el tema anterior conducen a un tercer tipo de reflexión, centrado precisamente en lo que realiza la institución escolar para adaptarse a este nuevo contexto social y qué modelos de escuelas comienzan a perfilarse. En este campo de estudios adquiere relevancia la discusión bibliográfica en torno a los distintos tipos de enfoques que abordan la diversidad, en el que destacan la aproximación asimilacionista, multicultural e intercultural (Barrios \& Palau, 2014; Carrillo, 2014; Stefoni, Stang \& Riedemann, 2016), así como la discusión en torno a los conceptos de integración e inclusión (Hernández, 2016; Mondaca, Rivera \& Gajardo, 2014). Un tema interesante en esta línea es la relación que se establece con el Programa de Educación intercultural Bilingüe implementado por el Estado de Chile, y las posibilidades de desarrollar proyectos educativos interculturales.

Los trabajos de Jiménez y Fardella profundizan en los distintos modelos de gestión de la diversidad presentes en la bibliografía especializada (Jiménez, 2014; Jiménez \& Fardella, 2015). Los autores identifican: i) la escuela segregacionista, en la que se establecen sistemas diferenciados para distintos grupos sociales, como por ejemplo género, religión, origen nacional y alumnos con algún tipo de diferencia que los hace "no aptos" para continuar en el sistema tradicional, ii) la escuela asimilacionista, con dos momentos o variaciones. Por un parte, una visión universalista, que incorpora a todos los alumnos bajo la creencia de la superioridad de la cultura hegemónica y que se plasma en un currículo uniforme para todos, sin atender a los requerimientos específicos de grupos particulares, y un segundo momento en el que el reconocimiento de ciertas diferencias se aborda desde una lógica compensatoria, es decir, asumiendo que grupos minoritarios presentarían ciertos hándicaps que deben ser nivelados; iii) escuela multicultural, en la que se pone el acento en el reconocimiento, el respeto a la diferencia y la interacción social; y iv) escuela intercultural, que cuestiona la esencialización cultural de la diversidad y la concentración del modelo anterior en sectores donde hay una alta población indígena o migrante; sin embargo, el aspecto más distintivo de este modelo es la propuesta transformadora de las relaciones de poder que sustentan la desigualdad entre grupos sociales.

Bajo esta tipología, la mayoría de los artículos revisados sostienen que el modelo de educación intercultural pareciera atender de mejor manera la diversidad. Este modelo, además, es coherente con lo que se ha planteado desde la política pública en Chile como educación inclusiva, superando algunas de las limitaciones que suponía el concepto de integración. Así, el documento sobre Orientaciones Técnicas para la inclusión educativa de estudiantes extranjeros (Mineduc, 2017) plantea que la valoración de la diversidad permite avanzar en la cohesión social, convivencia democrática y en una cultura de paz.

En los últimos años, nuestro país también ha avanzado en articular el principio de inclusión con objetivos de cohesión social, convivencia democrática y el fomento de una cultura de paz. En este sentido, las comunidades educativas que se reconocen desde la diversidad de las identidades que las componen, favorecen el diálogo intercultural, el aprendizaje de una convivencia que respeta y valora la diversidad y la construcción de marcos de participación que favorecen la expresión y representación de todas y todos" (MINEDUC. División de Educación General, Coordinación Nacional de Inclusión y Diversidad, 2017, p. 36). 
Más adelante, el mismo documento señala que el desafío de la inclusión es abordar aquellos mecanismos que producen exclusión y discriminación:

Trabajar en modo inclusivo significa comprender que la complejidad de estas transformaciones radica en identificar y abordar aquellos mecanismos que producen exclusión y discriminación, y que se encuentran asentados en las subjetividades de los actores y en la cultura institucional de las comunidades educativas. Estos mecanismos normalizadores que sostienen el referente de estudiantado homogéneo son los que hoy día requieren ser cuestionados" (MINEDUC. División de Educación General, Coordinación Nacional de Inclusión y Diversidad, 2017, p. 36).

El trabajo de campo que realizan las y los investigadores revisados respecto de este punto está centrado en observar y analizar las valoraciones de la diversidad, así como las prácticas implementadas en los establecimientos educacionales en relación con la migración (Bustos \& Gairin, 2017a; Cerón et al., 2017; Hernández, 2016; Jiménez \& Fardella, 2015; Mondaca et al., 2014; Moreno \& Oyarzún, 2013). Una de las conclusiones a la que llegan prácticamente todos los investigadores es la distancia entre el planteamiento teórico y declarativo de lo intercultural como modelo educativo, y la realidad que tienen los establecimientos educacionales.

Hernández (2016) por ejemplo, señala que los profesores consideran que ellos promueven la participación por igual en el aula, sin tomar en consideración el origen nacional de los estudiantes. Sin embargo, acota que no hay una prioridad por integrar esta diversidad de modo de transformarla en un recurso común para todo el estudiantado.

Los resultados obtenidos por esta investigación permiten concluir que los profesores en su quehacer profesional promueven la participación de todos los estudiantes al interior del aula, independiente de su nacionalidad. Bajo este principio de igualdad educativa, se otorgan las mismas oportunidades de aprendizaje para todos los niños y niñas; también se generan intercambios culturales entre los distintos grupos. En las clases, los estudiantes migrantes pueden difundir aspectos culturales de sus países; cuando esto sucede, los estudiantes chilenos manifiestan curiosidad y respeto por los saberes de los estudiantes migrantes. Aunque existen estos espacios de diálogo entre las otras culturas, se observa que no es prioridad integrar la diferencia cultural como un recurso que puede enriquecer los aprendizajes y la implementación del currículum (Hernández, 2016, p. 167).

Así, los resultados de la investigación lo llevan a concluir que las escuelas con un importante número de estudiantes migrantes no han logrado implementar una real política inclusiva que facilite la integración de los estudiantes, ni tampoco se observan planes y actividades concretas para llevarlo a cabo en el mediano o largo plazo.

\subsubsection{Racismo}

Los estudios evidencian las dificultades para avanzar en una inclusión real de la población migrante, así como en un modelo de escuela intercultural que sea capaz de transformar la posición de privilegio de la que goza la cultura dominante y terminar con la inferiorización de la cultura y de los grupos de inmigrantes. 
La discusión sobre este tema se desarrolla a partir de la comprensión sobre el racismo en las escuelas (Pavéz, 2012; Riedemann \& Stefoni, 2015; Suárez, 2015; Tijoux, 2013; Tijoux-Merino, 2013) y la construcción constante del discurso nacionalista hegemónico. Ambos argumentos están interrelacionados, pero una distinción analítica permite observar algunas distinciones importantes para tener en consideración.

Tijoux (2013), acertadamente, introduce el marco poscolonial para comprender las continuidades en la construcción racializada de las poblaciones en América Latina desde la llegada de los españoles al continente, en adelante. Indio y negro han sido de esta forma los sujetos dominados o aniquilados por los conquistadores, quienes a su vez se han definido a sí mismos como blancos y poseedores de la verdad y la razón.

En América Latina y en Chile esta alteridad se ha personificado en la figura del indio y del negro que debe ser conquistado o aniquilado. En nuestro país, durante el siglo XIX, este otro convertido en enemigo se ubicaba en el sur y se forjaba en la guerra contra el colonizador; hecho producido en un momento de higienización y de blanqueamiento, característicos de los procesos modernizadores ligados a políticas de migración selectiva destinadas a inmigrantes europeos que vinieron a poblar las tierras del sur (p. 290).

La jerarquía racial que ha permanecido en la base de las desigualdades sociales (Quijano, 2007) ha permitido también la construcción de un relato nacional negador de aquello que se ha inferiorizado. La idea de esta nación se ha construido sobre el imaginario de un pueblo blanco, homogéneo en términos culturales, heteronormativo y con un orden de género tradicional y conservador. De este modo, racismo y nacionalismo sirven como ejes estructurantes para situar a la migración en un lugar no sólo de subordinación, sino externo a aquello definido como la nación propia. Racismo y nacionalismo se superponen como matrices de significación para situar al migrante como alguien permanentemente alterizado y como alguien constantemente desvalorizado, mecanismo que sirve a su vez para facilitar los procesos de explotación laboral y económica que tiene lugar hoy en día.

Esta matriz presente en la sociedad chilena (y en el resto de América Latina) se reactiva y reproduce en la escuela a partir de la llegada de inmigrantes latinoamericanos. Los trabajos de investigación constatan la presencia de una ideología racista que sustenta estructuras de significado a partir de las cuales se definen relaciones sociales, representaciones y estereotipos. Los estudios también dan cuenta de que la escuela ha sido uno de los espacios privilegiados para la construcción del proyecto nacional, de ahí la doble dificultad de desmontar o deconstruir estos discursos nacionalistas y racistas, ya que ello se encuentra de alguna manera inscrito en el corazón del proyecto escolar. Los conceptos de inclusión e interculturalidad en su variante funcional (Tubino, 2005; Walsh, 2009) no bastan para transformar esta realidad. Las políticas de buena convivencia, de no ir acompañadas de una reflexión más profunda en torno a las condiciones de desigualdad que sustentan la discriminación, no logran resolver las tensiones que se instalan dentro de los establecimientos.

Para avanzar en una educación antirracista, algunos autores han elaborado guías pedagógicas con este objetivo en mente. Esto por cuanto la educación antirracista supone en primer lugar adquirir conciencia de las situaciones de privilegio en las que se encuentra aquellos pertenecientes a una cultura dominante, para luego generar prácticas transformadoras (Stang, Riedemann, Garcés \& Stefoni, 2017). 


\subsubsection{Buenas prácticas y experiencias}

Un último grupo de trabajos ha buscado rescatar iniciativas y prácticas implementadas en algunas escuelas del país. La selección de estas prácticas está orientada una vez más por el principio de interculturalidad (Donoso et al., 2009; Hirmas \& Blanco, 2009; Marín, 2014; Stang et al., 2017), que es entendido como el reconocimiento mutuo, la interacción a partir de condiciones de igualdad que descentran la posición de privilegio de quien históricamente se ha situado en el centro.

Uno de los casos documentados como buena práctica fue la iniciativa generada en una escuela en Santiago Centro, en la que se desarrolló una clase denominada "Historia, geografía y ciencias sociales de Suramérica". Se trató de una innovación curricular que tuvo como objetivo discutir procesos de integración de las distintas comunidades escolares a partir de ejes comunes en torno a la identidad suramericana (Alvites \& Jiménez, 2011; Donoso et al., 2009).

Otra de las prácticas documentadas es la experiencia de un taller de Arteterapia (Aynilab) realizado con hijos de migrantes, quienes a través del arte desarrollaron un espacio de convivencia e integración. Los trabajos realizados por niños y niñas en el norte de Chile permitieron comprender las condiciones de riesgo psicosocial a las que están expuestos, así como sus estrategias para abordarlas y elaborar relatos significativos para ellos (Marín, 2014).

\section{CONCLUSIONES}

La recopilación de estos artículos y trabajos de investigación da cuenta del desarrollo de un campo de investigación específico que se construye en la intersección entre los movimientos migratorios y los crecientes desafíos del sistema escolar.

En efecto, el sistema escolar atraviesa hoy una de las mayores transformaciones desde el proceso de privatización y mercantilización impulsado en dictadura. Se trata de la recuperación de la educación pública y el fin a la segregación y exclusión escolar, a partir de la implementación de la ley de inclusión. Junto a esto, parte importante de las escuelas se vuelven culturalmente más heterogéneas con la misión de generar cohesión social al mismo tiempo que respecto a la diversidad y aprendizajes de alta calidad, desafío que requieren cambios globales tanto en la formación de los docentes como también en la visión que las escuelas han construido de sí mismas dentro de la sociedad chilena.

La ley de inclusión ha dado pie a grandes discusiones en torno al sistema escolar, sin embargo, la situación de alumnos inmigrantes se ha mantenido fuera del corazón de la reforma y de la discusión política. Solo de manera reciente -y principalmente a través de la Ley de Convivencia- la presencia de alumnos inmigrantes comienza a ser abordada como un desafío para las escuelas del país. Uno de los problemas, sin embargo, que advertimos es que esta incorporación se realiza desde la mirada de la buena convivencia y no como un eje central de la ley de inclusión, ni tampoco como un eje de la discusión curricular. De hecho, hasta la fecha tampoco se ha aprovechado el espacio que la formación en ciudadanía comienza a ocupar en los establecimientos educativos.

Esto lleva a que quede en manos de los establecimientos, sus equipos directivos y profesores el diseño e implementación de proyectos interculturales. Insistimos en que la 
perspectiva que se desprende desde las propuestas que entrega el Ministerio de Educación (y que no son demasiadas) ponen el foco en la necesidad de garantizar una buena convivencia, pero no profundizan en la forma de revertir las relaciones de poder sobre las que se construyen las relaciones sociales entre los distintos grupos.

Una segunda reflexión que nos deja la revisión de la bibliografía refiere a las ausencias que se observan en la elaboración de preguntas y objetivos de la investigación. Al respecto, una de las primeras ausencias, tal como lo indicamos en el apartado metodológico, se relaciona con la necesidad de investigaciones en las que se amplíen los criterios muestrales, de modo de poner en juego nuevas variables hasta ahora muy presentes en los estudios sobre desigualdad, pero menos consideradas en estudios vinculados con migración. Un segundo elemento necesario de puntualizar es la relativa ausencia de trabajos en este campo que provengan desde la propia disciplina de educación. La mayoría de las investigaciones han sido realizadas por sociólogos, antropólogos, historiadores, entre otros, pero falta una gran discusión respecto de cómo la multiculturalidad se inserta también en el currículum escolar, en la didáctica, en las pedagogías de enseñanza. Esto requiere un conocimiento mucho más específico y propio de la tradición investigativa en educación. Un tercer elemento ausente dice relación con ciertos actores que no han sido suficientemente abordados en las investigaciones, tales como los diseñadores de políticas públicas, autoridades políticas en el ámbito de la educación, dirigentes estudiantiles, y la comunidad educacional ampliada. Finalmente, un cuarto aspecto es la importancia de incorporar una visión más amplia sobre niños y niñas migrantes, que incorpore experiencias de vida más allá de lo escolar, de modo de conocer sus trayectorias, lugares de origen, experiencias en cuanto hijos, compañeros, etc. Consideramos que, en la mayoría de los casos, la niñez migrante se encuentra reducida al rol de estudiantado, omitiendo una serie de dimensiones que hacen parte de la construcción de los sujetos, pero que no han sido suficientemente abordadas, con excepción del trabajo de Iskra Pavéz (Pavéz, 2012; 2013; 2017).

\section{REFERENCIAS BIBLIOGRÁFICAS}

\section{ARTÍCULOS Y CAPÍTULOS DE LIBRO REVISADOS PARA ESTE ESTADO DEL ARTE}

Alvites, L. M. \& Jiménez, R. (2011). Niños y niñas migrantes, desafío pendiente. Innovación educativa en escuela de Santiago de Chile. Synergies Chili, 7, 121-136.

Barrios, L., \& Palau, B. (2014). Educación intercultural en Chile: la integración del alumnado extranjero en el sistema escolar. educ educ, 17(3), 405-426.

Bravo, R. (2012). Inmigrantes en la escuela chilena: ciertas representaciones para ciertas políticas en educación. Revista Latinoamericana de educación inclusiva, 6(1), 39-52.

Bustos, R. \& Gairin, J. (2017a). Adaptación académica de estudiantes migrantes en contextos de fronteras. Calidad en la educación, 46, 193-220.

Bustos, R. \& Gairin, J. (2017b). Expectativas académicas de estudiantes y padres migrantes: el caso de Arica en la frontera de Chile y Perú. Revista Dilemas Contemporáneos: Educación, políticas y valores, $I V(3)$.

Carrillo, C. (2014). Reflexión en torno a las construcciones simbólicas de los/las profesores/as respecto de la «inclusión educativa» de hjos e hijas de inmigrantes en el sistema educativo chileno. Cuadernos de Trabajo Social, 11, 43-56.

Cerón, L., Alvarado, M. \& Poblete, R. (2017). Percepciones docentes en torno a la presencia de 
niños y niñas migrantes en escuelas de Santiago: Retos y desafíos para la inclusión. Revista Latinoamericana de educación inclusiva, 11(2), 233-246.

Contreras, D., Cortés, S. \& Fabio, C. (2012). Niños, niñas y adolescentes migrantes y su derecho a la educación en Chile. En ACNUR, OIM, UNICEF (Ed.), Los derechos de los niños, niñas $y$ adolescentes migrantes, refugiados y víctimas de trata internacional en Chile. Avances y desafíos. Santiago, Chile: ACNUR, OIM, UNICEF.

Donoso, A., Mardones, P. \& Contreras, R. (2009). Propuestas y desafíos a partir de la experiencia de una escuela con migrantes en el barrio Yungay, Santiago de Chile. Docencia, 37, 59-62.

Hernández, A. (2016). El currículo en contextos de estudiantes migrantes: Las complejidades del desarrollo curricular desde la perspectiva de los docentes de aula. Estudios Pedagógicos, $X L I I(2), 151-169$.

Hirmas, C. \& Blanco, R. (2009). Educar en la diversidad cultural: lecciones aprenidas desde la experiencia en América Latina. Docencia, 37.

Jiménez, F. (2014). Modelos de gestión de la diversidad para la escolarización del alumnado inmigrante en las escuelas chilenas: desafíos para la interculturalidad. Estudios Pedagógicos, $X L(2), 409-426$.

Jiménez, F., Aguilera, M., Valdés, R. \& Hernández, M. (2017). Migración y escuela: Análisis documental en torno a la incorporación de inmigrantes al sistema educativo chileno. Psicoperspectivas: Individuo y Sociedad, 16(1), 105-116.

Jiménez, F. \& Fardella, C. (2015). Diversidad y rol de la escuela. Discursos del profesorado en contextos educativos multiculturales en clave migratoria. Revista Mexicana de Investigación Educativa, 20(65), 419-441.

Joiko, S. \& Vásquez, A. (2016). Acceso y elección escolar de familias migrantes en Chile: No tuve problemas porque la escuela es abierta, porque acepta muchas nacionalidades. Calidad en la educación, 45.

Londoño, O., Maldonado, F. \& Calderón, L. (2014). Guía para construir estados del arte. International Corporation of Networks of Knowledge.

Marín, J. (2014). Ayni: Por una infanciasin fronteras. Arteterapia con hijos de migrantes en el norte de Chile. Arteterapia: Papeles de arteterapia y educación artística para la inclusión social, 9, 61-72.

MINEDUC. División de Educación General, Coordinación Nacional de Inclusión y Diversidad. (2017). Orientaciones técnicas para la inclusión educativa de estudiantes extranjeros. MINEDUC.

Mondaca, C., Rivera, P. \& Gajardo, Y. (2014). Educación parvularia e inclusión en el norte de Chile. Formando pequeños chilenos en las auas de Tarapacá. Alpha, 39, 251-266.

Moreno, K. \& Oyarzún, C. (2013). ¿Interculturalidad en el sistema educacional chileno?: Representaciones sociales de la integración de estudiantes exranjeros en dos establecimientos educacionalesmunicipales. Revista electrónica de psicología Istacalaca, 16(3).

Pavéz, I. (2012). Inmigración y racismo: experiencias de la niñez peruana en Santiago de Chile. Si Somos Americanos. revista de Estudios Transfronterizos, XII(1), 75-99.

. (2013). La infancia como sujeto de las políticas públicas e intervenciones sociales: el caso de la niñez migrante en el Chile del siglo XXI. En Acta Científica del XXIX Congreso de la Asociación Latinoamericana de Sociología. Santiago, Chile.

. (2017). Integración sociocultural y derechos de las niñas y los niños migrantes en el contexto local. El caso de Recoleta, Región Metropolitana, Chile. Chungara, Revista de Antropología Chilena, 49(4).

Poblete, R. \& Galaz, C. (2016). Niños y niñas migrantes. Trayectorias de inclusión educativa en escuelas de la Región Metropolitana. Superintendencia de Educación. Chile.

(2017). Aperturas y cierres para la inclusión educativa de niños/as migrantes en Chile. Estudios Pedagógicos, XLIII(3), 239-257.

Quijano, A. (2007). Coloniality and modernity/rationality. Cultural studies, 21(2-3), 168-178.

Riedemann, A. \& Stefoni, C. (2015). Sobre el racismo, su negación, y las consecuencias para una educación anti-racista en la enseñanza secundaria chilena. Polis, Revista Latinoamericana, 42. 
Salas, N., Del Río, M. F., Kong, F. \& San Martín, C. (2016). Caracterización y prejuicio acerca de los inmigrantes en el sistema escolar. Informe Proyecto FONIDE F911424.

Salas, N., Kong, F. \& Gazmuri, R. (2017). La investigación socio territorial: una propuesta para comprender los procesos de inclusión de los migrantes en las escuelas. Revista Latinoamericana de educación inclusiva, 11(1), 73-91.

Sánchez, A., Navas, L. \& Holgado, P. (2013). Inmigración y educación intercultural en la formación inicial docente. Estudios Pedagógicos, XXXIX(1), 239-251.

Sleeter, C., Montecinos, C. \& Jiménez, F. (2016). Preparing teachers for social justice in the context of education policies that deepen class segregation in schools: the case of Chile. En J. Lamper \& B. Burnett (Eds.), Theacher education for high poverty schools (pp. 171-191). Switzerland: Springer.

Stang, F., Riedemann, A., Garcés, A. \& Stefoni, C. (2017). Guía pedagógica para una educación intercultural anti-racista y con perspectiva de género. Ideas, experiencias y herramientas. Santiago, Chile.

Stefoni, C., Acosta, E., Gaymer, M. \& Casas-Cordero, F. (2010). El derecho a la educación de los niños y niñas inmigrantes en Chile. Editorial: Universidad de Deusto.

Stefoni, C., Stang, F., \& Riedemann, A. (2016). Educación e interculturalidad en Chile: un marco para el análisis. Estudios Internacionales, 185, 153-182.

Suárez, D. (2015). Nuevos migrantes, viejos racismos: Los mapas parlantes y la niñez migrante e Chile. Revista Latinoamericana de Ciencias Sociales, Niñez y Juventud, 13(12), 627-643.

Tijoux, M. E. (2013). Las escuelas de la inmigración en la ciudad de Santiago: Elementos para una educación contra el racismo. Polis (Santiago), 12(35), 287-307. https://doi.org/10.4067/S071865682013000200013.

Tijoux-Merino, M. E. (2013). Niños(as) marcados por la inmigración peruana: estigma, sufrimientos, resistencias. Convergencia. Reviista de Ciencias Sociales, 20(61), 83-104.

\section{OTRAS REFERENCIAS BIBLIOGRÁFICAS}

Ceriani, P. (2011). Luces y sombras en la legislación migratoria latinoamericana. Nueva Sociedad, (233).

Cerruti, M., Maguid, A. \& Díaz Gil, A. (2011). Migrantes Sudamericanos en España: Panorama y Políticas. Cuadernos Migratorios No 1. OIM.

Guizardi, M., Moraga, J. \& Garcés, A. (2014). Los procesos migratorios actuales en contextos latinoamericanos: nuevos itinerarios y reconfiguración de controles fronterizos. Revista de Estudios Sociales 35(48), 177-183. https://doi.org/10.7440/res48.2014.14.

Quijano, A. (2007). Colonialidad del poder y clasificación social. En S. Castro Gómez \& S. Grosfogel (Eds.), El giro decolonial. Reflexiones para una diversidad epistémica más allá del capitalismo global (pp. 93-126). Bogotá, Colombia: Siglo del hombre editores. Instituto de Estudios Sociales Contemporáneos y Universidad Javeriana.

Rojas, N. \& Silva, C. (2016). La migración en Chile: Breve reporte y caracterización. Observatorio Iberoamericano sobre Movilidad Humana. Migración y Desarrollo, OBIMID.

Texidó, E. \& Gurrieri. (2012). Panorama migratorio de América del Sur. OIM (Perfiles Migratorios). Buenos Aires, Argentina: OIM.

Stefoni, C. (2011). Perfil Migratorio de Chile. OIM.

Tubino, F. (2005). La praxis de la interculturalidad en los Estados nacionales latinoamericanos. Cuadernos Interculturales, 3(5), 83-96.

Walsh, C. (2009). Interculturalidad crítica y educación intercultural. Presentado en Interculturalidad y educación Intercultural. Organizado por el Instituto Internacional de integración del Convenio Andrés Bello, La Paz, Bolivia. 
